\title{
Is innate immunity our best weapon for flattening the curve?
}

\author{
Leonard Angka, ${ }^{1,2}$ Marisa Market, ${ }^{1,2}$ Michele Ardolino, ${ }^{1,2,3}$ and Rebecca C. Auer ${ }^{1,4}$ \\ 'Cancer Therapeutics Program, Ottawa Hospital Research Institute, Ottawa, Ontario, Canada. ${ }^{2}$ Department of Biochemistry, Microbiology, and Immunology, ${ }^{3}$ Centre for Infection, Immunity, and Inflammation, \\ and ${ }^{4}$ Division of Ceneral Surgery, Department of Surgery, University of Ottawa, Ottawa, Ontario, Canada.
}

he COVID-19 pandemic is a stern reminder not to take our immune system for granted. The fact that some individuals contract and clear the SARS-CoV-2 virus without apparent symptoms stands in sharp contrast to the damage that this virus has brought upon more vulnerable populations, including the elderly and patients with chronic conditions or cancer. While the differences in severity of infection between these populations are multifactorial, it is likely that innate immunity provides the underpinning, given its central role in the early response to viral infections. Within two decades, there have been three known coronavirus zoonoses (SARS-CoV-1, MERS, and SARS-CoV-2), all of which have taken a devastating toll on the human and economic health of affected societies. Unfortunately, with the frequency and diffusion of novel zoonoses, this is unlikely to be our last battle. As we begin the long and daunting recovery from this pandemic, we must take the opportunity to think about how to exploit our innate immune system to better prepare us to fight the next virus.

\section{Harnessing innate immunity "One may know how to conquer without being able to do it" (1).}

In most countries, interventions have been put in place to flatten the curve. The goal of these interventions is to slow the spread of the virus so that capacity of medical resources, such as hospital beds, ventilators, and personal protective equipment, is not exceeded and we can adequately care for the sick. While social distancing has proven highly effective in flattening the curve, it is often impossible for essential workers or vulnerable patients. Moreover, the ripple effects of social distancing on our economy and well-being are not sustainable long term.
Ideally, alternative strategies to social distancing should (a) still protect highrisk individuals, (b) accelerate the global recovery, and (c) easily be adapted to tackle future pandemics. Here, we move forward the hypothesis that harnessing innate immunity is our best weapon for fighting novel viral outbreaks by preventing a productive infection or by accelerating viral clearance.

Active immunization provides protection against a specific vaccination target through antigen-specific responses by adaptive lymphocytes. An effective antigen-specific vaccine can completely protect the majority of healthy individuals, so it is not surprising that the world is waiting for this panacea with bated breath. It is important to remember, however, that human endemic coronaviruses, which are responsible for up to $35 \%$ of common colds, often reinfect individuals, sometimes even in the same year (2), despite $90 \%$ of individuals developing coronavirus-specific antibodies (3). Even when infection yields high antibody titers, the titer half-life is, in some cases, short (4).

Further undermining the hope for an effective respiratory/coronavirus vaccine are examples from veterinary medicine, where coronaviruses are scourges that have eluded eradication despite vaccination. This is surely not for a lack of trying, as bovine coronaviruses cause several diseases, including shipping fever: a deadly respiratory disease common in commercial feedlots that is responsible for an estimated loss of $\$ 900$ million annually (5). Another example is the highly lethal feline coronavirus, which shares similarities to SARS-CoV-1/2, including viral persistence, induction of profound lympho- penia, and a protracted clinical course (6). These similarities should raise a cautionary flag, since humoral responses against this feline coronavirus, rather than being protective, result in a more severe disease course. In these cases, subneutralizing levels of spike $S$ protein antibodies opsonize the virus and promote infection of immune cells expressing Fc receptors (antibody-dependent enhancement of disease). It is likely that current vaccine endeavors will overcome these barriers, but this will take time and the vaccine may be ineffective against future coronaviruses (7).

An intriguing alternate strategy to vaccination relies on enhancing innate immunity, which has been successfully adopted in veterinary medicine to prevent the transmission of respiratory viruses in cattle housed in close quarters (8). Specifically, administration of an inactivated Parapoxvirus ovis virus broadly activates innate immune components, including dendritic cells, NK cells, and type I IFNs, and protects livestock from many respiratory illnesses unrelated to Parapoxvirus.

Innate immunity acts as our body's front-line troops. Pattern-recognition receptors recognize viral components and prompt IFN production. IFNs have direct antiviral activity and choreograph the innate and adaptive lymphocyte responses. They are so central to antiviral responses that zoonotic coronaviruses dedicate much of their genome to suppressing them (9), lending credence to the hypothesis that prophylactic or early IFN treatment will circumvent immune evasion and promote viral clearance (10). Based on this rationale, clinical trials with recombinant IFNs are underway. One clinical trial (ClinicalTrials.gov NCT04320238) is using nasally administered recombinant IFN- $\alpha 1 \beta$ to prevent SARS-CoV- 2 infection among medical workers. A second clinical trial (NCT04293887) will administer IFN$\alpha 1 \beta$ to newly diagnosed patients within 7 
days of symptoms onset, while a third trial (NCT04331899) will treat mild COVID-19 cases with type III IFN, which has partially overlapping functions, but reduced toxicity compared with type I IFN. If successful, these trials will provide the proof of concept that prophylactic/early stimulation of the innate immune system provides protection against SARS-CoV-2 and, in theory, against future coronaviruses.

\section{Training innate immunity \\ "He who is prudent and lies in wait for an enemy who is not, will be victorious" (1).}

Like any army, our innate immune system will respond more effectively when primed. Recent studies have highlighted how the adaptive immune response as well as innate immunity can be trained to respond to pathogenic insults. Trained immunity refers to a more potent inflammatory response caused by long-lasting epigenetic modifications in myeloid progenitors (11). The lack of specificity of the secondary response may be the key to successfully exploiting trained immunity against COVID-19 and future pandemics.

The bacille Calmette-Guérin (BCG, live attenuated Mycobacterium bovis) vaccine was developed against tuberculosis and in many countries is part of a child's immunization schedule. Epidemiological studies have shown that BCG vaccination is associated with an approximately $30 \%-40 \%$ reduction in unrelated respiratory infections and reduced infectious mortality in childhood (12). Corroboration for this effect was obtained in a randomized controlled trial where BCG vaccination protected against experimental infection of an attenuated yellow fever virus (13). Accordingly, there were fewer confirmed COVID-19 cases and deaths in countries with mandatory BCG vaccination (14), although a contrasting report was recently published (15), highlighting that more studies are required to clarify whether BCG vaccination confers long-term protection. However, given the abundance of mechanistic evidence showing that heterologous vaccination results in trained immunity, BCG immunization is currently being explored in the Netherlands (NCT04328441), Australia (NCT04327206), Egypt (NCT04350931), and the USA (NCT04348370) in an attempt to protect health care workers against COVID-19. Unfortunately, BCG is contraindicated in many vulnerable patients, such as those undergoing chemotherapy. However, the IMM-101 vaccine (heat-killed Mycobacterium obuense) has a mechanism of action similar to that of BCG, with an established safety profile in cancer patients (16). The Canadian Cancer Trials Group has proposed testing IMM101 in a clinical trial to protect high-risk cancer patients who cannot self-isolate due to their treatment (CCTG ID IC8).

When considering the mechanism of action of IFNs and heterologous vaccines, NK cells emerge as important mediators of the therapeutic response. NK cells are key components of the antiviral response due to their ability to eliminate virally infected cells and produce antiviral cytokines upon activation (17). NK cell function is heavily dependent on the cytokine milieu that these cells act on, and both IFNs and other proinflammatory cytokines produced by trained myeloid cells can prime and boost NK antiviral functions. Viral infections, particularly with cytomegalovirus, generate a subset of memory NK cells that respond more potently to a secondary exposure to the pathogen (18). Although NK memory responses have not been studied in the context of coronavirus infections, therapeutic strategies that promote beneficial inflammation could leverage the additional benefits provided by the establishment of a memory NK cell pool.

\section{Timing becomes the key determinant}

"He will win who knows when to fight

and when not to fight" (1).

If an early and strong innate immune response is the key to protection and effective viral clearance, why do reports of innate immune dysregulation leading to severe pathophysiology dominate the clinical SARS-CoV literature? Initially, immune suppression may be required to establish a productive infection. This has been confirmed in hospitalized patients in whom severe symptomatic illness was associated with innate immune suppression (19). Clinical studies have shown that coronavirus infections reduce the number of circulating NK cells and alter their functional status. NK cells in patients infected with coronaviruses are less functional, indicating a reduced ability to eliminate infected cells, and present higher expression of inhibitory receptors, e.g., NKG2A, which increases the threshold of activation for NK cells (19). However, in more severe COVID-19 infections, myeloid cells accumulate in the lungs and promote the recruitment and activation of lymphocytes, including NK cells. Once in the lungs, NK cells are likely to be among the main producers of IFN- $\gamma$, which contributes to immunopathology and the development of acute respiratory distress syndrome (20).

The discordance in our assertions that innate immunity eradicates COVID-19 while having a pathological role in severe disease can be explained by whom we study and when we study them. Most correlative clinical studies are undertaken in patients who are already hospitalized or critically ill because those are the ones accessible for blood samples, while mildly symptomatic or asymptomatic patients have not been properly studied because they remain at home in self-quarantine. Murine studies, which are highly controlled, will therefore be very informative when contemplating when to therapeutically boost innate immune responses (21). A case in point is that IFN treatment within 6 hours after infection was curative in mice infected with SARS-COV-1, whereas delayed administration, at 12 hours, was ineffective (22), revealing that timing is a key determinant of efficacy.

The data point to a model whereby prophylactic or early innate immune activation can prevent or attenuate the symptoms following COVID-19, but when ineffective, viral-induced innate immune dysfunction, followed by a dysregulated innate immune response, may worsen immunopathology and the severity of infection. We must take into account that innate immune cells, including myeloid and NK cells, have been implicated in immunopathology when therapeutically eliciting an antiviral response. Therefore, therapeutic interventions need to be effective and well timed, as an untimely administration of immunostimulating agents can exacerbate immunopathology and aggravate disease (23).

How then can we determine when to exploit immune activation, and in which patients? A simple and direct solution to this problem could once again come from 
innate immunity and particularly from the measurement of NK cell reactivity. NK cells' responsiveness is finely tuned by the inflammatory milieu, of which IFNs are an essential part. While it can be challenging to accurately assess the levels of different cytokines in COVID-19 patients, measuring NK cell responsiveness is straightforward. NK-Vue measures IFN- $\gamma$ released by NK cells stimulated with a proprietary stimulating cocktail and is currently used in cancer patients to assess NK cell activity before treatment (24). A similar concept could be translated to viral infections. Interestingly, NK cell activity is strikingly low in patients who are particularly vulnerable to COVID-19, such as the elderly, cancer patients, and patients recovering from surgery (24). This assay could therefore be used to identify patients at high risk of symptomatic and severe COVID-19 and those that would benefit from innate immune activation.

\section{Informing therapeutic interventions}

"Ifyou know the enemy and know yourself, you need not fear the result of a hundred battles" (1).

In addition to our efforts to find a vaccine against SARS-CoV-2 and to flatten the curve, we should endeavor to understand the biological and pathophysiological aspects of viral infection and of the immune response to the virus. Knowledge of the molecular, cellular, and systemic relationships between coronaviruses and the host's innate immune system will provide us with more resources to rationally and effectively fight this and future outbreaks. A focus on the innate immune system will allow translation of our understanding from one pandemic to another. If one lesson has to be learned from previous coronavirus outbreaks, it is that a chance has been missed to accumulate important knowledge that could have informed therapeutic interventions for the current pandemic. We believe that in the battle against this pandemic and the next, we should put our faith in the innate immune system after all, organisms have been doing it for over half a billion years.

\section{Acknowledgments}

Research in the Ardolino lab is funded by grants from the Canadian Institute for Health Research (CIHR), the Cancer Research Society (CRS), and the Joan Sealy Trust, and research in the Auer lab is funded by grants from CIHR, CRS, the Ontario Institute of Cancer Research, the Terry Fox Foundation, and BioCanRx.

Address correspondence to: Michele Ardolino, 501 Smyth Road, Cancer Center, 3-328, Ottawa, Ontario K1H 8M2, Canada. Phone: 613.737 .8899 ext. 77257; Email: m.ardolino@uottawa.ca.

1. Giles L. Sun Tzǔ on the Art of War: The Oldest Military Treatise in the World. London, United Kingdom: Luzac \& Company; 1910.

2. Galanti M, Shaman J. Direct observation of repeated infections with endemic coronaviruses [preprint]. Posted on medRxiv May 3, 2020. https://doi.org/10.1101/2020.04.27.20082032.

3. Severance EG, et al. Development of a nucleocapsid-based human coronavirus immunoassay and estimates of individuals exposed to coronavirus in a U.S. metropolitan population. Clin Vaccine Immunol. 2008;15(12):1805-1810.

4. Callow KA, Parry HF, Sergeant M, Tyrrell DA. The time course of the immune response to experimental coronavirus infection of man. Epidemiol Infect. 1990;105(2):435-446.

5. Johnson KK, Pendell DL. Market impacts of reducing the prevalence of bovine respiratory disease in United States beef cattle feedlots. Front Vet Sci. 2017;4:189.

6. de Groot-Mijnes JD, van Dun JM, van der Most RG, de Groot RJ. Natural history of a recurrent feline coronavirus infection and the role of cellular immunity in survival and disease. J Virol. 2005;79(2):1036-1044.

7. Amanat F, Krammer F. SARS-CoV-2 vaccines: status report. Immunity. 2020;52(4):583-589.

8. Apley M. Ancillary therapy of bovine respiratory disease. Vet Clin North Am Food Anim Pract. 1997;13(3):575-592.

9. Kindler E, Thiel V. To sense or not to sense viral RNA--essentials of coronavirus innate immune evasion. Curr Opin Microbiol. 2014;20:69-75.

10. Park A, Iwasaki A. Type I and type III Interferons - induction, signaling, evasion, application to combat COVID-19 [published online
May 27, 2020]. Cell Host Microbe. https://doi. org/10.1016/j.chom.2020.05.008.

11. Netea MG, et al. Trained immunity: a tool for reducing susceptibility to and the severity of SARS-CoV-2 infection. Cell. 2020;181(5):969-977.

12. Higgins JP, et al. Association of BCG, DTP, and measles containing vaccines with childhood mortality: systematic review. BMJ. 2016;355:15170.

13. Arts RJW, et al. BCG vaccination protects against experimental viral infection in humans through the induction of cytokines associated with trained immunity. Cell Host Microbe. 2018;23(1):89-100.e5

14. Miller A, Reandelar MJ, Fasciliogne K, Roumenova V, Li Y, Otazu GH. Correlation between universal BCG vaccination policy and reduced morbidity and mortality for COVID-19: an epidemiological study [preprint]. https://doi.org/ 10.1101/2020.03.24.20042937. Posted on medRxiv March 28, 2020.

15. Hamiel U, Kozer E, Youngster I. SARS-CoV-2 rates in BCG-vaccinated and unvaccinated young adults. JAMA. 2020;323(22):2340-2341.

16. Dalgleish AG, et al. Randomised, open-label, phase II study of gemcitabine with and without IMM-101 for advanced pancreatic cancer. $\mathrm{Br} J$ Cancer. 2016;115(7):789-796.

17. Jost S, Altfeld M. Control of human viral infections by natural killer cells. Annu Rev Immunol. 2013;31:163-194

18. Brillantes M, Beaulieu AM. Memory and memory-like NK cell responses to microbial pathogens. Front Cell Infect Microbiol. 2020;10:102.

19. Zheng M, et al. Functional exhaustion of antiviral lymphocytes in COVID-19 patients. Cell Mo Immunol. 2020;17(5):533-535.

20. Channappanavar R, Perlman S. Pathogenic human coronavirus infections: causes and consequences of cytokine storm and immunopathology. Semin Immunopathol. 2017;39(5):529-539.

21. Israelow B et al. Mouse model of SARSCoV-2 reveals inflammatory role of type I interferon signaling [preprint]. https://doi. org/10.1101/2020.05.27.118893. Posted on bioRxiv May 27, 2020.

22. Channappanavar R, et al. Dysregulated type I interferon and inflammatory monocyte-macrophage responses cause lethal pneumonia in SARS-CoV-infected mice. Cell Host Microbe. 2016;19(2):181-193.

23. Rajaram S, et al. The promise and peril of natural killer cell therapies in pulmonary infection. Immunity. 2020;52(6):887-889.

24. Angka L, et al. Natural killer cell IFN $\gamma$ secretion is profoundly suppressed following colorectal cancer surgery. Ann Surg Oncol. 2018;25(12):3747-3754. 